\title{
Association Between Obstructive Sleep Apnea and Reduced Mortality in Critically III Patients: A Propensity Score-Based Analysis
}

\section{Ping Lin \\ Xiaoqian Li \\ Jiarui Zhang \\ Zongan Liang}

Department of Respiratory and Critical Care Medicine, West China School of Medicine and West China Hospital, Sichuan University, Chengdu, 61004I, People's Republic of China
Correspondence: Zongan Liang Department of Respiratory and Critical Care Medicine, West China School of Medicine and West China Hospital, Sichuan University, Chengdu, 61004I, Sichuan, People's Republic of China Email20202324025198@stu.scu.edu.cn; liangza@scu.edu.cn
Background: The impact of obstructive sleep apnea (OSA) on the prognosis of intensive care unit (ICU) patients remains controversial. Thus, this study aimed to determine the association between OSA status and outcomes in ICU patients.

Methods: We conducted a retrospective cohort study composed of 38,177 critically ill patients from the Medical Information Mart for Intensive Care III (MIMIC-III) database. Propensity score matching was used to evaluate the association between OSA and mortality in ICU adults. Results: A total of 38,177 critically ill patients were included in this study. Among them, 1428 (3.71\%) patients had OSA. Before matching, patients with OSA had a significantly lower 28-day mortality (relative risk (RR), 0.47; 95\% confidence interval (CI), 0.38-0.58), 90-day mortality (RR, 0.50; 95\% CI, 0.43-0.60), ICU mortality (RR, 0.41; 95\% CI, 0.310.54 ), and in-hospital mortality (RR, 0.46 ; 95\% CI, 0.36-0.57). After adjusting with propensity-score matching, the findings of reduced risk of mortality remained unchanged. The RR of 28-day mortality, 90-day mortality, ICU mortality, in-hospital mortality was 0.57 (95\% CI, 0.34-0.57), 0.53 (95\% CI, 0.40-0.70), 0.54 (95\% CI, 0.37-0.79), and 0.55 (95\% CI, $0.39-0.77)$, respectively.

Conclusion: This study indicated that ICU patients with OSA had a significantly lower risk of mortality compared with those without OSA.

Keywords: obstructive sleep apnea, MIMIC-III, intensive care units, mortality

\section{Introduction}

Obstructive sleep apnea (OSA) is a highly prevalent breathing disorder, characterized by frequent episodes of complete or partial upper airway collapse during sleep, resulting in episodic hypoxemia and arousal. ${ }^{1,2}$ The overall prevalence of OSA ranges from $3 \%$ to $17 \%$ in the general adult population, and advancing age, male sex, and higher body-mass index increase OSA prevalence. ${ }^{3-6}$ Growing evidence indicates that OSA is an independent risk factor for various diseases, including hypertension, diabetes, venous thromboembolism, and stroke. ${ }^{7-9}$

Recently, studies are being focused on the association between OSA status and the prognosis of critically ill patients, but with controversial results having been published in two previous studies. ${ }^{10,11}$ One found that OSA was associated with decreased mortality, ${ }^{11}$ but the other did not identify such benefits. ${ }^{10}$ The lack of body mass index data and small sample size undermined the reliability of the above studies. Therefore, a large wellplanned study was needed to determine the association between OSA and outcomes in critically ill patients. 
In this article, we conducted a retrospective cohort study by using data derived from the Medical Information Mart for Intensive Care III (MIMIC-III) database. ${ }^{12}$ The MIMIC-III database is an extensive, public database compromising comprehensive clinical data, which could provide sufficient information to determine the impact of OSA on the prognosis of critically ill patients in the intensive care unit (ICU).

\section{Methods}

\section{Data Source}

The present study was based on MIMIC-III, a large, freely available database of clinical data of more than 40,000 ICU patients admitted to the Beth Israel Deaconess Medical Center between 2001 and 2012. ${ }^{12,13}$ We were allowed to access the database by the Institutional Review Boards of the Massachusetts Institute of Technology and the Beth Israel Deaconess Medical Center (researchers certification number 9266789).

\section{Inclusion and Exclusion Criteria}

Adult patients (aged 18 years or above) were included in this study. Critically ill patients diagnosed with OSA were extracted from the Medical Information Mart for MIMIC-III database using the International Classification of Diseases- (ICD-) 9 codes (code = "32,723"). For patients who were admitted to the ICU more than once, only data related to the first ICU admission was considered.

\section{Data Extraction}

The following information was extracted from the database using structured query language (SQL) with PostgreSQL (version 12): patient demographics, laboratory test results, comorbidities, and other clinical variables. Demographic characteristics included age, sex, weight, simplified acute physiology score II (SAPS II), and sequential organ failure assessment score (SOFA); The comorbidities included hypertension, coronary artery disease, chronic obstructive pulmonary disease, diabetes mellitus, and chronic kidney disease. Laboratory measurements included hemoglobin, white blood cell (WBC), platelets, total bilirubin, creatinine, and lactate. The primary outcome was 28-day and 90-day mortality. The secondary outcome included ICU mortality, inhospital mortality, and the length of ICU stay.

\section{Statistical Analysis}

Continuous variables were expressed as median and interquartile range, and categorical variables were shown by number and percentage. Continuous variables were compared by the Wilcoxon rank-sum test, and categorical variables were compared by chi-square or Fisher's exact test. Propensity score matching was used to adjust for confounding factors. ${ }^{14,15}$ The survival curves were plotted by the Kaplan-Meier method and compared by the Log rank test. ${ }^{16}$ All statistical analyses were performed using Stata software (version 14.0, USA). A p $<0.05$ was considered statistically significant.

\section{Results}

This retrospective cohort study included patients 18 years of age or older. The patient flowchart is shown in Figure 1. A total of 38,177 critically ill patients were included in the study after reviewing the MIMIC-III database. Of the study cohort, $1428(3.71 \%)$ patients had OSA, and the remaining 36,749 patients did not have OSA. Table 1 shows the baseline characteristics for patients with and without OSA. Patients in the OSA group were younger, more likely to be men, more likely to have lower SAPS II scores and higher weight, and more likely to have hypertension, coronary artery disease, chronic obstructive pulmonary disease, and chronic kidney disease. Before matching, the OSA group had a significantly lower 28day mortality (RR, 0.47 ; $95 \%$ CI,0.38-0.58), 90-day mortality (RR, $0.50 ; 95 \%$ CI,0.43-0.60), ICU mortality (RR,0.41; 95\% CI,0.31-0.54), in-hospital mortality (RR,0.46; 95\% CI,0.36-0.57) (Table 1).

\section{Propensity Score Analysis}

The 402 patients with OSA were matched to 402 patients without OSA by propensity score matching. After adjusting with propensity-score matching, these imbalances were all eliminated (1:1 matching ratio). After matching, the findings of reduced risk of mortality remained unchanged. The RR of 28-day mortality, 90-day mortality, ICU mortality, in-hospital mortality was 0.57 (95\% CI, $0.34-0.57), 0.53$ (95\% CI, 0.40-0.70), 0.54 (95\% CI, $0.37-0.79)$, and 0.55 (95\% CI, 0.39-0.77), respectively (Table 2). Figure 2 shows the Kaplan-Meier survival estimates for patients with and without OSA. Patients with OSA had a better prognosis than those without OSA. There was no difference between the groups in the length of ICU stay before and after matching (Table 2). 


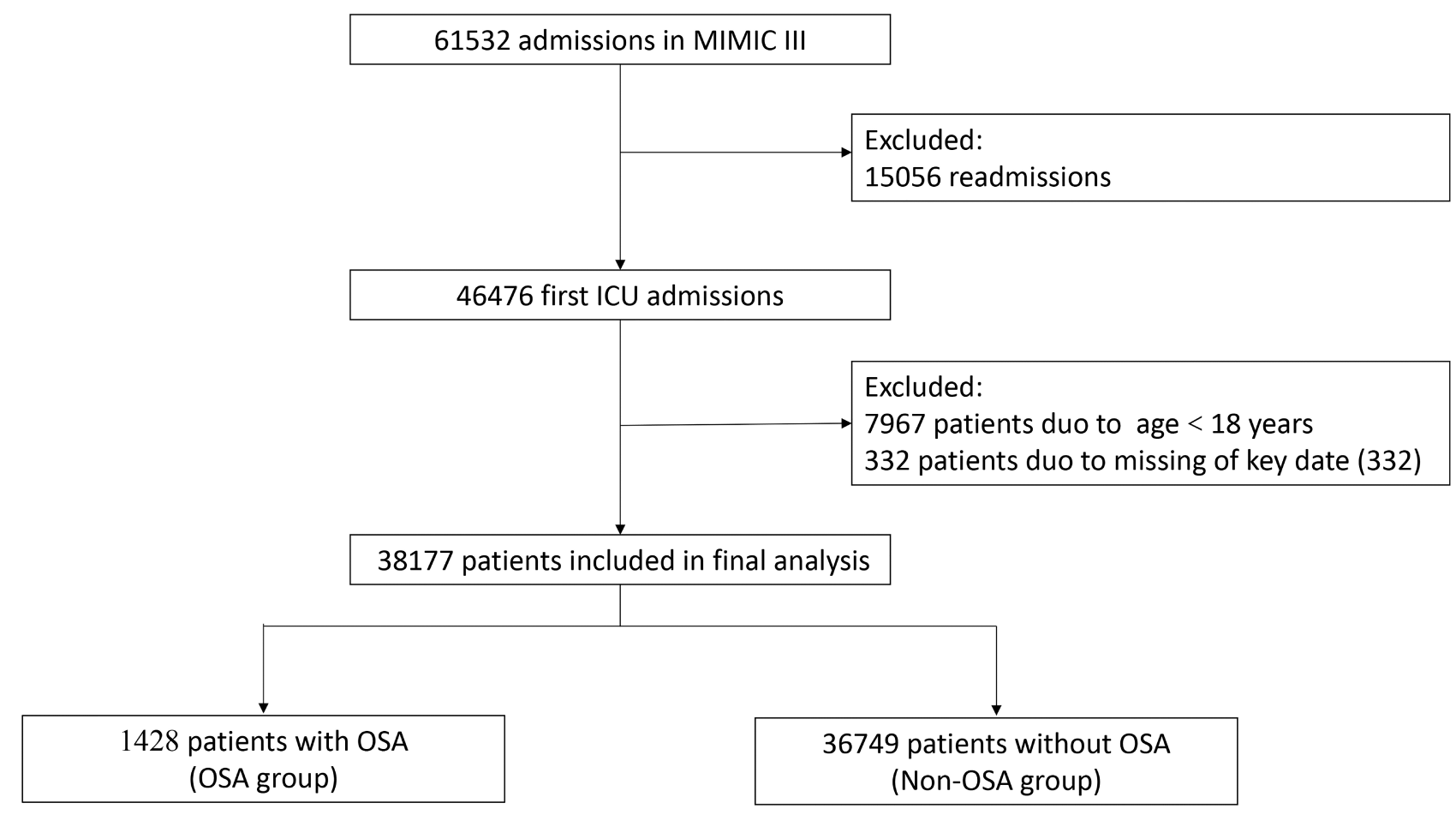

Figure I Flowchart of patient selection.

\section{Mortality in Older Patients}

Previous studies showed a decline of relative mortality in sleep apnea patients after the age of $50 .{ }^{17}$ In this study, we also calculated the mortality in older patients (age $>50$ years) with and without OSA. Before matching, older OSA patients had a significantly lower 28-day mortality $(14.38 \%$ vs $6.59 \%, \mathrm{P}<0.001)$, 90-day mortality $(20.34 \%$ vs $9.67 \%$, $\mathrm{P}<0.001)$, ICU mortality ( $8.81 \%$ vs $3.51 \%, \mathrm{P}<0.001)$, inhospital mortality ( $12.35 \%$ vs $5.57 \%, \mathrm{P}<0.001)$. After matching, older OSA patients was still associated with lower 28-day mortality $(21.97 \%$ vs $12.73 \%, \mathrm{P}=0.006)$, 90-day mortality $(32.17 \%$ vs $18.32 \%, \mathrm{P}<0.001)$, ICU mortality $(17.52 \%$ vs $10.25 \%, \quad \mathrm{P}=0.008)$, in-hospital mortality (21.02\% vs $12.73 \%, \mathrm{P}=0.005)$.

\section{Discussion}

We studied 38,177 ICU patients to determine the relationships between OSA and all-cause mortality. We found that compared with those without OSA, patients with OSA had a significantly lower risk of 28-, 90-, ICU- and in-hospital mortality. After adjusting with confounding factors by propensity score matching, OSA was still associated with better survival in ICU patients.
OSA is not uncommon in critically ill patients, especially in patients with acute hypercapnic respiratory failure. ${ }^{18,19}$ One might wonder whether OSA affects the prognosis of critically ill patients. In patients with subarachnoid hemorrhage, OSA was found to be associated with decreased risk of in-hospital mortality $(7.3 \%$ to $11.5 \%, \mathrm{P}=$ $0.003) .{ }^{20}$ Similar results $(2.4 \%$ vs $6.2 \%)$ were also reported in critically ill patients admitted to the ICU ward in Bolona et al study. ${ }^{11}$ However, Bolona's study was flawed by lacking weight data, so they did not figure out whether the low mortality rate is caused by obesity or OSA itself. Moreover, this observation was not supported by subsequent research demonstrating that OSA had no impact on ICU mortality. ${ }^{10}$ Therefore, the impact of OSA on the outcomes of ICU patients remains controversial. The current study might provide credible information about the impact of OSA on the prognosis of ICU patients based on a large sample size. We found that compared with patients without OSA, patients with OSA had lower risks of mortality in unadjusted analyses. After adjusted for age, gender, weight, SAPS II scores, sequential organ failure assessment (SOFA) score, comorbidities, and laboratory tests, OSA was still associated with decreased risk of mortality, which indicated that OSA itself might be a protective factor for mortality. 
Table I Patient Baseline Demographic and Clinical Characteristics

\begin{tabular}{|c|c|c|c|}
\hline Characteristic & Without OSA $(\mathrm{N}=36,749)$ & With OSA $(N=1428)$ & $P$ value \\
\hline \multicolumn{4}{|l|}{ Baseline characteristics } \\
\hline Age(y) & $65.82[52.34,78.1 \mathrm{I}]$ & $63.04[53.54,71.51]$ & $<0.001$ \\
\hline Man, n (\%) & $20,650(56.19)$ & $959(67.16)$ & $<0.001$ \\
\hline Weight(kg) & $77.30[65.20,91.00]$ & $104.00[86.60,123.70]$ & $<0.001$ \\
\hline SAPS II & $33.00[24.00,42.00]$ & $31.00[23.00,39.00]$ & $<0.001$ \\
\hline SOFA score & $3.00[2.00,5.00]$ & $4.00[2.00,5.50]$ & 0.191 \\
\hline \multicolumn{4}{|l|}{ Comorbidities } \\
\hline Hypertension, n (\%) & $\mid 5,875(43.20)$ & $746(53.50)$ & $<0.001$ \\
\hline CAD, n (\%) & $10,385(28.26)$ & $460(32.21)$ & 0.001 \\
\hline COPD, n (\%) & $712(1.94)$ & $64(4.48)$ & $<0.001$ \\
\hline Diabetes mellitus, n (\%) & $9227(25.11)$ & $634(44.40)$ & $<0.001$ \\
\hline CKD, n (\%) & $4026(10.96)$ & $279(19.54)$ & $<0.001$ \\
\hline \multicolumn{4}{|l|}{ Laboratory tests } \\
\hline Hemoglobin $(g / d L)$ & $10.80[9.50,12.30]$ & II.10[9.60,12.70] & $<0.001$ \\
\hline WBC(K/ul) & $10.80[7.90,14.60]$ & $10.50[7.70,14.00]$ & 0.031 \\
\hline Platelets(K/ul) & $200.00[146.00,266.00]$ & $208.00[157.00,269.00]$ & $<0.001$ \\
\hline Total bilirubin(mg/dl) & $0.70[0.40,1.40]$ & $0.60[0.40,1.10]$ & $<0.001$ \\
\hline Creatinine $(\mathrm{mg} / \mathrm{dl})$ & $0.90[0.70,1.30]$ & $1.00[0.80,1.40]$ & $<0.001$ \\
\hline Lactate $(\mathrm{mmol} / \mathrm{L})$ & $1.80[1.20,2.80]$ & $1.60[1.10,2.40]$ & $<0.001$ \\
\hline \multicolumn{4}{|l|}{ Clinical outcomes } \\
\hline 28-day mortality, n (\%) & $46 \mid 4(12.56)$ & $84(5.88)$ & $<0.001$ \\
\hline 90-day mortality, n (\%) & $6494(17.67)$ & $127(8.89)$ & $<0.001$ \\
\hline ICU mortality, n (\%) & $2902(7.90)$ & $46(3.22)$ & $<0.001$ \\
\hline In-hospital mortality, n (\%) & $4013(10.92)$ & $71(4.97)$ & $<0.001$ \\
\hline Length of ICU stay (day) & $2.11[1.20,4.10]$ & $2.08[1.22,4.11]$ & 0.694 \\
\hline
\end{tabular}

Note: Continuous variables were expressed as median and interquartile range.

Abbreviations: OSA, obstructive sleep apnea; SAPSII, simplified acute physiology score II; SOFA, sequential organ failure assessment; CAD, coronary artery disease; COPD, chronic obstructive pulmonary disease; CKD, chronic kidney disease; WBC, white blood cell; ICU, intensive care unit.

Although in general OSA was associated with a worse long-term prognosis, this study did not show an increase of relative mortality in critically ill patients. There were several possible mechanisms related to the protective effect of OSA in critical care patients. First, in our study, patients without OSA were older and had higher SAPS II scores, indicated that patients without OSA suffered greater severity of illness. Second, long-term adaptation to hypoxia might cause ischemic preconditioning due to the nocturnal cycles of hypoxia-reoxygenation, which might alleviate the acute stress response and protect the cardiocirculatory system against infarction and further ischemic insults. ${ }^{21}$ Furthermore, Berger et al found that the mobilization, proliferative and angiogenic capacities of endothelial progenitor cells were heightened in patients with OSA, indicating that vascular endothelial cell repair might also be involved in the mechanism of cardiovascular protection. ${ }^{22}$ Another possible mechanism by which OSA might reduce mortality was the development of coronary collaterals. It was known that oxygen consumption and resting energy expenditure were increased significantly in critically ill patients. ${ }^{23}$ OSA might promote angiogenesis through oxidative stress and upregulation of vascular endothelial growth factor in patients with coronary artery disease, which might trigger the development of coronary collaterals and improve blood supply to the heart. ${ }^{24}$ This adaptive change in the cardiovascular system might increase myocardial oxygen and reduce cardiovascular risk among patients with serious illnesses. ${ }^{25}$ Studies had reported that patients with OSA and intermittent hypoxemia during sleep had less severe cardiac injury during an acute myocardial infarction compared with those without OSA. ${ }^{26}$ Future studies are warranted to further elucidate the specific mechanism because limited evidences support these hypotheses described above. ${ }^{27}$ 
Table 2 Propensity Score-Matched Patients with and without OSA

\begin{tabular}{|c|c|c|c|}
\hline Characteristic & Without OSA(N=402) & With OSA $(\mathrm{N}=402)$ & $P$ value \\
\hline \multicolumn{4}{|l|}{ Baseline characteristics } \\
\hline Age(y) & $62.75[51.71,72.36]$ & $62.90[53.22,70.93]$ & 0.767 \\
\hline Man, n (\%) & $280(69.65)$ & $273(67.91)$ & 0.594 \\
\hline Weight(kg) & $104.00[86.20,127.30]$ & $108.00[89.90,127.00]$ & 0.191 \\
\hline SAPS II & $37.00[29.00,47.00]$ & $38.00[29.00,48.00]$ & 0.449 \\
\hline SOFA score & $5.00[3.00,8.00]$ & $5.00[3.00,8.00]$ & 0.518 \\
\hline \multicolumn{4}{|l|}{ Comorbidities } \\
\hline Hypertension, n (\%) & $209(51.99)$ & $196(48.76)$ & 0.359 \\
\hline CAD, n (\%) & $130(32.34)$ & $110(27.36)$ & 0.123 \\
\hline COPD, n (\%) & $16(3.98)$ & $17(4.23)$ & 0.859 \\
\hline Diabetes mellitus, n (\%) & $212(52.74)$ & $198(49.25)$ & 0.323 \\
\hline CKD, n (\%) & $83(20.65)$ & $85(21.14)$ & 0.862 \\
\hline \multicolumn{4}{|l|}{ Laboratory tests } \\
\hline Hemoglobin (g/dL) & $10.70[9.40,12.20]$ & $11.00[9.60,12.60]$ & 0.189 \\
\hline WBC(K/ul) & $11.15[7.80,15.40]$ & $10.95[7.40,15.60]$ & 0.548 \\
\hline Platelets(K/ul) & $203.50[143.00,265.00]$ & $198.00[148.00,271.00]$ & 0.520 \\
\hline Total bilirubin(mg/dl) & $0.70[0.40,1.10]$ & $0.60[0.40,1.20]$ & 0.320 \\
\hline Creatinine(mg/dl) & $1.15[0.80,1.80]$ & I.I0[0.80, I.70] & 0.276 \\
\hline Lactate (mmol/L) & $1.60[1.20,2.60]$ & $1.60[1.10,2.50]$ & 0.116 \\
\hline \multicolumn{4}{|l|}{ Clinical outcomes } \\
\hline 28-day mortality, n (\%) & $82(20.40)$ & $47(11.69)$ & 0.001 \\
\hline 90-day mortality, n (\%) & $117(29.10)$ & $62(15.42)$ & $<0.001$ \\
\hline ICU mortality, n (\%) & $67(16.67)$ & $36(8.96)$ & 0.001 \\
\hline In-hospital mortality, n (\%) & $80(19.90)$ & $44(10.95)$ & $<0.001$ \\
\hline Length of ICU stay (day) & $5.23[2.79,11.10]$ & $4.97[2.45,10.54]$ & 0.522 \\
\hline
\end{tabular}

Note: Continuous variables were expressed as median and interquartile range.

Abbreviations: OSA, obstructive sleep apnea; SAPSII, simplified acute physiology score II; SOFA, sequential organ failure assessment; CAD, coronary artery disease; COPD, chronic obstructive pulmonary disease; CKD, chronic kidney disease; WBC, white blood cell; ICU, intensive care unit.

Our study had some limitations. First, our study was a retrospective observational study, which made it subject to confounding. Although we used propensity score matching to balance potential confounding factors, residual confounding could not be fully ruled out. Second, this was a single-center study, which might limit the
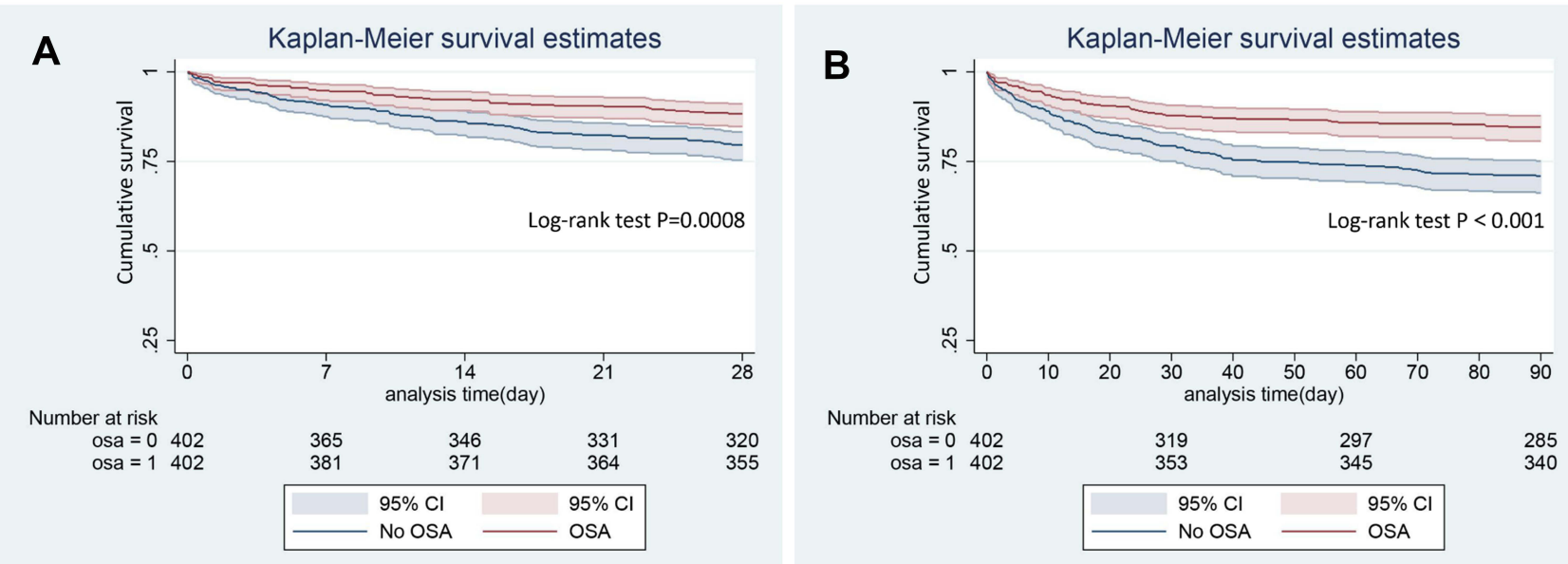

Figure 2 Kaplan-Meier curve (and 95\% confidence limits) depicts predicted overall survival between OSA and No OSA groups. (A) 28-day follow up; (B) 90-day follow up. 
generalizability of our results. Third, all OSA diagnoses were based on ICD-9 codes. Therefore, we did not conduct subgroup analyses according to OSA severity due to a lack of detailed data on polysomnography.

\section{Conclusions}

In conclusion, our results showed that OSA was associated with decreased risk of mortality in critically ill patients, independently of weight and comorbidities. The potential mechanism related to a better prognosis of OSA needs to be further studied.

\section{Ethics}

The MIMIC-III database is a freely used critical care medical database. Access to the database for research was approved by the Institutional Review Boards of the Massachusetts Institute of Technology and the Beth Israel Deaconess Medical Center (researchers certification number 9266789).

\section{Funding}

This study was funded by the National Key Research and Development Program of China (Grant 2016YFC1 304303).

\section{Disclosure}

The authors report no conflicts of interest in this work.

\section{References}

1. Punjabi NM. The epidemiology of adult obstructive sleep apnea. Proc Am Thorac Soc. 2008;5(2):136-143. doi:10.1513/pats.200709-155MG

2. Gottlieb DJ, Punjabi NM. Diagnosis and management of obstructive sleep apnea: a review. JAMA. 2020;323(14):1389-1400. doi:10.1001/ jama.2020.3514

3. Senaratna CV, Perret JL, Lodge CJ, et al. Prevalence of obstructive sleep apnea in the general population: a systematic review. Sleep Med Rev. 2017;34:70-81. PubMed PMID: 27568340. doi:10.1016/j. smrv.2016.07.002

4. Tufik S, Santos-Silva R, Taddei JA, Bittencourt LRA. Obstructive sleep apnea syndrome in the Sao Paulo Epidemiologic Sleep Study. Sleep Med. 2010;11(5):441-446. doi:10.1016/j.sleep.2009.10.005

5. Reddy EV, Kadhiravan T, Mishra HK, et al. Prevalence and risk factors of obstructive sleep apnea among middle-aged urban Indians: a Community-Based Study. Sleep Med. 2009;10(8):913-918. doi:10.1016/j.sleep.2008.08.011

6. Veasey SC, Rosen IM. Obstructive sleep apnea in adults. $N$ Engl $J$ Med. 2019;380(15):1442-1449. Epub 2019/ 04/11. PubMed PMID: 30970189. doi:10.1056/NEJMcp1816152

7. Zamarron C, García Paz V, Riveiro A. Obstructive sleep apnea syndrome is a systemic disease. current evidence. Eur J Intern Med. 2008;19(6):390-398. Epub 2008/ 10/14. PubMed PMID: 18848171. doi:10.1016/j.ejim.2007.12.006
8. Reutrakul S, Mokhlesi B. Obstructive sleep apnea and diabetes: a state of the art review. Chest. 2017;152(5):1070-1086. doi:10.1016/j.chest.2017.05.009

9. Alonso-Fernández A, Toledo-Pons N, García-Río F. Obstructive sleep apnea and venous thromboembolism: overview of an emerging relationship. Sleep Med Rev. 2020;50:101233. Epub 2019/ 12/16. PubMed PMID: 31838272. doi:10.1016/j.smrv.2019.101233

10. Bailly S, Galerneau LM, Ruckly S, et al. Impact of obstructive sleep apnea on the obesity paradox in critically ill patients. J Crit Care. 2020;56:120-124. Epub 2020/ 01/04. PubMed PMID: 31896445. doi:10.1016/j.jcrc.2019.12.016

11. Bolona E, Hahn PY, Afessa B. Intensive care unit and hospital mortality in patients with obstructive sleep apnea. J Crit Care. 2015;30(1):178-180. Epub 2014/ 12/03. PubMed PMID: 25457113. doi:10.1016/j.jcrc.2014.10.001

12. Johnson AE, Pollard TJ, Shen L, et al. MIMIC-III, a freely accessible critical care database. Sci Data. 2016;3(1):160035. Epub 2016/ 05/25. PubMed PMID: 27219127; PubMed Central PMCID: PMCPMC4878278. doi:10.1038/sdata.2016.35

13. Goldberger AL, Amaral LA, Glass L, et al. PhysioBank, PhysioToolkit, and PhysioNet: components of a new research resource for complex physiologic signals. Circulation. 2000;101 (23):E215-20. Epub 2000/ 06/14. PubMed PMID: 10851218. doi:10.1161/01.cir.101.23.e215

14. Austin PC, Fine JP. Propensity-score matching with competing risks in survival analysis. Stat Med. 2019;38(5):751-777. Epub 2018/ 10/ 23. PubMed PMID: 30347461; PubMed Central PMCID: PMCPMC6900780. doi:10.1002/sim.8008

15. Benedetto U, Head SJ, Angelini GD, Blackstone EH. Statistical primer: propensity score matching and its alternatives. Eur J Cardiothorac Surg. 2018;53(6):1112-1117. Epub 2018/ 04/24. PubMed PMID: 29684154. doi:10.1093/ejcts/ezy167

16. Jager KJ, Van Dijk PC, Zoccali C, Dekker FW. The analysis of survival data: the Kaplan-Meier method. Kidney Int. 2008;74 (5):560-565. doi:10.1038/ki.2008.217

17. Lavie P, Lavie L, Herer P. All-cause mortality in males with sleep apnoea syndrome: declining mortality rates with age. Eur Respir J. 2005;25(3):514-520. Epub 2005/ 03/02. PubMed PMID: 15738297. doi:10.1183/09031936.05.00051504

18. Thille AW, Córdoba-Izquierdo A, Maitre B, Boyer L, Brochard L, Drouot X. High prevalence of sleep apnea syndrome in patients admitted to ICU for acute hypercapnic respiratory failure: a Preliminary Study. Intensive Care Med. 2018;44(2):267-269. Epub 2017/ 11/22. PubMed PMID: 29159561. doi:10.1007/s00134017-4998-3

19. Dou L, Lan H, Reynolds DJ, et al. Association between obstructive sleep apnea and acute kidney injury in critically ill patients: a Propensity-Matched Study. Nephron. 2017;135(2):137-146. doi:10.1159/000453367

20. Kaculini C, Wallace DJ, Haywood AE, et al. Protective effects of obstructive sleep apnea on outcomes after subarachnoid hemorrhage: a nationwide analysis. Neurosurgery. 2020;87 (5):1008-1015. Epub 2020/ 06/17. PubMed PMID: 32542358. doi:10.1093/neuros/nyaa242

21. Lavie L, Lavie P. Ischemic preconditioning as a possible explanation for the age decline relative mortality in sleep apnea. Med Hypotheses. 2006;66(6):1069-1073. doi:10.1016/j.mehy.2005.10.033

22. Berger S, Aronson D, Lavie P, Lavie L. Endothelial progenitor cells in acute myocardial infarction and sleep-disordered breathing. $\mathrm{Am}$ J Respir Crit Care Med. 2013;187(1):90-98. Epub 2012/ 11/17. PubMed PMID: 23155141. doi:10.1164/rccm.201206-1144OC

23. Moriyama S, Okamoto K, Tabira Y, et al. Evaluation of oxygen consumption and resting energy expenditure in critically ill patients with systemic inflammatory response syndrome. Crit Care Med. 1999;27(10):2133-2136. Epub 1999/ 11/05. PubMed PMID: 10548194. doi:10.1097/00003246-199910000-00009 
24. Steiner S, Schueller PO, Schulze V, Strauer BE. Occurrence of coronary collateral vessels in patients with sleep apnea and total coronary occlusion. Chest. 2010;137(3):516-520. Epub 2009/ 10/28. PubMed PMID: 19858231. doi:10.1378/chest.091136

25. Lavie L, Lavie P. Coronary collateral circulation in sleep apnea: a cardioprotective mechanism? Chest. 2010;137(3):511-512.Epub 2010/ 03/06. PubMed PMID: 20202945. doi:10.1378/chest.092657
26. Shah N, Redline S, Yaggi HK, et al. Obstructive sleep apnea and acute myocardial infarction severity: ischemic preconditioning? Sleep Breath. 2013;17(2):819-826. Epub 2012/ 10/24. PubMed PMID: 23090861. doi:10.1007/s11325-012-0770-7

27. Lavie L. Oxidative stress in obstructive sleep apnea and intermittent hypoxia-revisited-the bad ugly and good: implications to the heart and brain. Sleep Med Rev. 2015;20:27-45. Epub 2014/ 08/27. PubMed PMID: 25155182. doi:10.1016/j.smrv.2014.07.003

\section{Publish your work in this journal}

The International Journal of General Medicine is an international, peer-reviewed open-access journal that focuses on general and internal medicine, pathogenesis, epidemiology, diagnosis, monitoring and treatment protocols. The journal is characterized by the rapid reporting of reviews, original research and clinical studies across all disease areas. The manuscript management system is completely online and includes a very quick and fair peer-review system, which is all easy to use. Visit http://www.dovepress.com/ testimonials.php to read real quotes from published authors.

Submit your manuscript here: https://www.dovepress.com/international-journal-of-general-medicine-journal 